\title{
Impact of dopant compensation on the deactivation of boron-oxygen recombination centers in crystalline silicon
}

\author{
Bianca Lim, ${ }^{1, a)}$ An Liu, ${ }^{2}$ Daniel Macdonald, ${ }^{2}$ Karsten Bothe, ${ }^{1}$ and Jan Schmidt ${ }^{1}$ \\ ${ }_{1}^{1}$ Institut für Solarenergieforschung Hameln (ISFH), Am Ohrberg 1, D-31860 Emmerthal, Germany \\ ${ }^{2}$ School of Engineering, College of Engineering and Computer Science, The Australian National University, \\ Canberra ACT 0200, Australia
}

(Received 16 September 2009; accepted 19 November 2009; published online 10 December 2009)

\begin{abstract}
The boron-oxygen recombination center responsible for the light-induced degradation of Czochralski silicon solar cells can be permanently deactivated by illumination at elevated temperature. In this study, we examine the impact of dopant compensation on the deactivation process. The experimental results show that the deactivation rate depends inversely on the total boron concentration instead of the net doping concentration, suggesting that boron is directly involved in the deactivation process. A linear dependence of the activation energy on the total boron concentration further supports this conclusion. (C) 2009 American Institute of Physics.
\end{abstract}

[doi:10.1063/1.3272918]

Recombination-active boron-oxygen complexes form in boron-doped Czochralski-grown silicon $(\mathrm{Cz}-\mathrm{Si})$ under illumination at room temperature. These complexes typically limit the carrier lifetime in oxygen-rich silicon. ${ }^{1}$ As a consequence, solar cells fabricated on B-doped Cz-Si suffer from a loss in energy conversion efficiency of up to $10 \%$ relative., ${ }^{2,3}$ A permanent deactivation of the defect can be achieved through illumination at elevated temperature. ${ }^{4-6}$ The deactivation process was found to be thermally activated and activation energies between 0.61 (Refs. 4 and 5) and $0.71 \mathrm{eV}$ (Ref. 6) have been reported.

In this work, we present carrier lifetime measurements aimed at investigating the impact of dopant compensation on the deactivation process and find that the deactivation rate is inversely proportional to the total boron concentration. In addition, a linear dependence of the activation energy on the total boron concentration is revealed. Both results provide strong evidence that boron plays a role in the deactivation process.

We examine $\mathrm{Cz}-\mathrm{Si}$ wafers from five different ingots, three are exclusively doped with boron (noncompensated controls) and two ingots are doped with boron and phosphorus. The resistivities $\rho$ of the noncompensated reference wafers are $5.8,1.26$, and $0.45 \Omega \mathrm{cm}$, as measured by the four-point-probe method after thermal donor annihilation.
The resistivities of the compensated samples are 1.20 and $0.52 \Omega \mathrm{cm}$, respectively.

Using the model of Klaassen ${ }^{7,8}$ (assuming a temperature of $25^{\circ} \mathrm{C}$ ), we subsequently determine the hole mobility $\mu_{\mathrm{hK}}$ and the equilibrium hole concentration $p_{0 \rho}$ in the noncompensated samples via a self-consistent calculation. The obtained values are summarized in Table. I. The hole concentrations in the wafers are additionally determined by electrochemical capacitance voltage (ECV) measurements ${ }^{9}$ and we find very good agreement between $p_{0 \mathrm{ECV}}$ and $p_{0 \rho}$ (see Table I).

To determine the boron $\left(N_{\mathrm{A}}\right)$ and the phosphorus concentrations $\left(N_{\mathrm{D}}\right)$ in the compensated samples, we use a method introduced by Macdonald et al., ${ }^{10}$ which is based on the measurement of the association time constant $\tau_{\text {assoc }}$ of iron-boron $(\mathrm{FeB})$ pairs. Since no interstitial iron can be detected in the as-grown samples, we transfer iron from $\mathrm{Fe}$ contaminated multicrystalline silicon wafers during a hightemperature anneal at $1000{ }^{\circ} \mathrm{C}$ to the monocrystalline $\mathrm{Cz}-\mathrm{Si}$ wafers. ${ }^{11}$ The same measurements are also performed on the noncompensated wafers, where the hole concentration $p_{0}$ equals the acceptor concentration $N_{\mathrm{A}}$, i.e., the density of substitutional boron. As can be seen from Table I, for the noncompensated samples the values of $N_{\mathrm{A}}$ from the $\tau_{\text {assoc }}$

TABLE I. Resistivities $\rho$ as measured by the four-point-probe method, hole mobilities $\mu_{\mathrm{hK}}$ in the noncompensated wafers from Klaassen's model, equilibrium hole concentration $p_{0 \rho}$ determined from $\rho$ and $\mu_{\mathrm{hK}}$, equilibrium hole concentration $p_{0 \mathrm{ECV}}$ determined via ECV measurements, and the acceptor concentration $N_{\mathrm{A}}$ measured via the iron-acceptor repairing time constants $\tau_{\text {assoc. }}$.

\begin{tabular}{|c|c|c|c|c|c|}
\hline Wafer & $\begin{array}{l}\rho \text { from four-point-probe } \\
{[\Omega \mathrm{cm}]}\end{array}$ & $\begin{array}{c}\text { Hole mobility } \\
\mu_{\mathrm{hK}}(\text { Klaassen }) \\
{\left[\mathrm{cm}^{2} / \mathrm{V} \mathrm{s}\right]}\end{array}$ & $\begin{array}{c}p_{0 \rho} \text { from } \rho \text { and } \mu_{\mathrm{hK}} \\
{\left[\mathrm{cm}^{-3}\right]}\end{array}$ & $\begin{array}{c}p_{0 \mathrm{ECV}} \text { from ECV } \\
{\left[\mathrm{cm}^{-3}\right]}\end{array}$ & $\begin{array}{c}N_{\mathrm{A}} \text { from } \tau_{\text {assoc }} \\
\quad\left[\mathrm{cm}^{-3}\right]\end{array}$ \\
\hline 74 (noncompensated) & 5.8 & 462 & $2.33 \times 10^{15}$ & $\cdots$ & $3.37 \times 10^{15}$ \\
\hline 72 (noncompensated) & 1.26 & 431 & $1.15 \times 10^{16}$ & $1.14 \times 10^{16}$ & $1.85 \times 10^{16}$ \\
\hline 73 (noncompensated) & 0.45 & 384 & $3.61 \times 10^{16}$ & $3.8 \times 10^{16}$ & $3.35 \times 10^{16}$ \\
\hline 45 (compensated) & 1.20 & $\cdots$ & $\cdots$ & $1.5 \times 10^{16}$ & $3.0 \times 10^{16}$ \\
\hline 44 (compensated) & 0.52 & $\cdots$ & $\cdots$ & $4.1 \times 10^{16}$ & $9.2 \times 10^{16}$ \\
\hline
\end{tabular}

${ }^{a)}$ Electronic mail: lim@isfh.de. 
TABLE II. Resistivities $\rho$ of the compensated samples as measured by the four-point-probe method, equilibrium hole concentration $p_{0 \mathrm{ECV}}$ determined via ECV measurements, acceptor concentration $N_{\mathrm{A}}$ measured via the iron-acceptor repairing time constants $\tau_{\text {assoc }}$, phosphorus concentration $N_{\mathrm{D}}=N_{\mathrm{A}}-p_{0 \mathrm{ECV}}$, hole mobilities $\mu_{\mathrm{hK}}$ from Klaassen's model using $N_{\mathrm{A}}$ and $N_{\mathrm{D}}$, measured hole mobilities $\mu_{\mathrm{h}} \equiv\left(\begin{array}{lll}\rho & e & p_{0 \mathrm{ECV}}\end{array}\right)^{-1}$, equilibrium hole concentration $p_{0 \rho} \equiv\left(\begin{array}{lll}\rho & e & \mu_{\mathrm{hK}}\end{array}\right)^{-1}$, and the compensation level $C=\left(N_{\mathrm{A}}+N_{\mathrm{D}}\right) /\left(N_{\mathrm{A}}-N_{\mathrm{D}}\right)$.

\begin{tabular}{|c|c|c|c|c|c|c|c|c|}
\hline Wafer & $\begin{array}{c}\rho \text { from } \\
\text { four-point-probe } \\
{[\Omega \mathrm{cm}]}\end{array}$ & $\begin{array}{c}p_{0 \mathrm{ECV}} \text { from ECV } \\
{\left[\mathrm{cm}^{-3}\right]}\end{array}$ & $\begin{array}{c}N_{\mathrm{A}} \text { from } \tau_{\text {assoc }} \\
\quad\left[\mathrm{cm}^{-3}\right]\end{array}$ & $\begin{array}{c}N_{\mathrm{D}}=N_{\mathrm{A}}-p_{0 \mathrm{ECV}} \\
{\left[\mathrm{cm}^{-3}\right]}\end{array}$ & $\begin{array}{c}\text { Hole mobility } \\
\mu_{\mathrm{hK}}(\text { Klaassen }) \\
{\left[\mathrm{cm}^{2} / \mathrm{V} \mathrm{s}\right]}\end{array}$ & $\begin{array}{c}\text { Measured } \mu_{\mathrm{h}} \\
{\left[\mathrm{cm}^{2} / \mathrm{V} \mathrm{s}\right]}\end{array}$ & $\begin{array}{c}p_{0 \rho} \text { from } \rho \\
\text { and } \mu_{\mathrm{hK}} \\
{\left[\mathrm{cm}^{-3}\right]}\end{array}$ & $C=\frac{N_{\mathrm{A}}+N_{\mathrm{D}}}{N_{\mathrm{A}}-N_{\mathrm{D}}}$ \\
\hline 45 (compensated) & 1.20 & $1.5 \times 10^{16}$ & $3.0 \times 10^{16}$ & $1.5 \times 10^{16}$ & 380 & 347 & $1.37 \times 10^{16}$ & 3 \\
\hline 44 (compensated) & 0.52 & $4.1 \times 10^{16}$ & $9.2 \times 10^{16}$ & $5.1 \times 10^{16}$ & 306 & 293 & $3.92 \times 10^{16}$ & 3.5 \\
\hline
\end{tabular}

measurements are in good agreement with the hole concentrations $p_{0 \mathrm{ECV}}$ and $p_{0 \rho}$.

Knowing the boron concentrations $N_{\mathrm{A}}$, the phosphorus concentrations $N_{\mathrm{D}}$ in the compensated samples then follow from $N_{\mathrm{D}}=N_{\mathrm{A}}-p_{0 \mathrm{ECV}}$ to be $1.5 \times 10^{16} \mathrm{~cm}^{-3}$ for the $1.20 \Omega \mathrm{cm}$ wafer and $5.1 \times 10^{16} \mathrm{~cm}^{-3}$ for the $0.52 \Omega \mathrm{cm}$ wafer, as summarized in Table II. The compensation level $C=\left(N_{\mathrm{A}}+N_{\mathrm{D}}\right) /\left(N_{\mathrm{A}}-N_{\mathrm{D}}\right)$ in the $0.52 \Omega \mathrm{cm}$ wafer is thus higher than in the $1.20 \Omega \mathrm{cm}$ sample (3.5 and 3, respectively). Looking at the measured hole mobility $\mu_{\mathrm{h}}$ $\equiv\left(\begin{array}{lll}\rho & e & p_{0 \mathrm{ECV}}\end{array}\right)^{-1}$, where $e$ is the elementary charge, we find a reduction in majority carrier mobility with an increase in compensation level $\left(\mu_{\mathrm{h}}=347 \mathrm{~cm}^{2} / \mathrm{Vs}\right.$ in the $1.20 \Omega \mathrm{cm}$ compensated wafer and $\mu_{\mathrm{h}}=293 \mathrm{~cm}^{2} / \mathrm{Vs}$ in the compensated $0.52 \Omega \mathrm{cm}$ material). This reduction is in good agreement with Klaassen's model, ${ }^{7,8}$ which yields $\mu_{\mathrm{hK}}$ $=380 \mathrm{~cm}^{2} / \mathrm{Vs}$ in the $1.20 \Omega \mathrm{cm}$ compensated material and $\mu_{\mathrm{hK}}=306 \mathrm{~cm}^{2} / \mathrm{Vs}$ in the $0.52 \Omega \mathrm{cm}$ samples. Table II also includes the hole concentrations $p_{0 \rho}$ as given by $p_{0 \rho}$ $\equiv\left(\begin{array}{lll}\rho & e & \mu_{\mathrm{hK}}\end{array}\right)^{-1}$, which are in very good agreement with the hole concentrations determined via ECV measurements.

For lifetime measurements the samples were cleaned and underwent a phosphorus-diffusion step to remove metal impurities. Prior to passivation with plasma-enhanced chemical vapor deposited silicon nitride, ${ }^{12}$ the $n^{+}$regions on both surfaces were removed by wet chemical etching.

Illumination at elevated temperature was performed on a hotplate with a halogen lamp. The light intensity was adjusted with a calibrated solar cell while the temperature was controlled by a proportional-integral-derivative-controller. All stated temperatures refer to the set temperature, leading to an error in the actual temperature of up to $5{ }^{\circ} \mathrm{C}$. Carrier lifetimes were measured at room temperature using the quasi-steady-state photoconductance technique. ${ }^{13}$

Samples from the five different ingots were cured at $185^{\circ} \mathrm{C}$ and at a light intensity of $100 \mathrm{~mW} / \mathrm{cm}^{2}$. Figure 1 shows the normalized defect concentration $N_{\mathrm{t}}^{*}$ of the $1.26 \Omega \mathrm{cm}$ noncompensated control and the $1.20 \Omega \mathrm{cm}$ compensated sample plotted against the duration of illumination $t . N_{\mathrm{t}}^{*}$ is defined as the difference of the inverse lifetime $\tau^{-1}(t)$ measured at time $t$ and the inverse initial lifetime measured before degradation $\tau_{0}^{-1}$, i.e., $N_{\mathrm{t}}^{*}=\tau^{-1}(t)-\tau_{0}^{-1}$. For better comparison, $N_{\text {t.max }}^{*}$ is furthermore normalized to one. The deactivation rate $R_{\mathrm{de}}$ is determined by fitting a curve of the form $N_{\mathrm{t}}^{*}(t)=A \times \exp \left(-R_{\mathrm{de}} t\right)+B$ to the measured data (solid lines in Fig. 1). The difference between the noncompensated wafer (filled green circles) and the compensated sample (open purple squares) in Fig. 1 is obvious; the deactivation in the compensated sample takes three times longer than in the noncompensated wafer. Comparing the $0.45 \Omega \mathrm{cm}$ control wafer and the $0.52 \Omega \mathrm{cm}$ compensated sample we find a similar decrease in the deactivation rate by a factor of 2.4 (not plotted).

The deactivation rates $R_{\text {de }}$ determined at $185^{\circ} \mathrm{C}$ and at a light-intensity of $100 \mathrm{~mW} / \mathrm{cm}^{2}$ are plotted versus the total boron concentration $N_{\mathrm{A}}$ in Fig. 2 (filled symbols). The double-logarithmic scale reveals a reciprocal dependence of the deactivation rate on the boron concentration for the noncompensated controls (filled blue triangles up). The fit yields a slope of -0.74 , i.e., $R_{\mathrm{de}} \sim N_{\mathrm{A}}^{-0.74}$. Plotting the deactivation rate of the compensated samples versus the hole concentration $p_{0}$ (open red triangles down) results in a considerable deviation from the fit. However, if the deactivation rate is plotted as a function of the total boron concentration $N_{\mathrm{A}}$ (filled red triangles down), there is good agreement with the fit to the data of the noncompensated wafers. The observed dependence of the deactivation rate $R_{\mathrm{de}}$ on the total boron concentration $N_{\mathrm{A}}$ in compensated silicon, in contrast to a dependence on the hole concentration $p_{0}=N_{\mathrm{A}}-N_{\mathrm{D}}$, is a strong indication that boron plays a role in the deactivation process. With regard to the proposed formation of boronphosphorus pairs in compensated silicon, ${ }^{14-16}$ this finding also implies that the involvement of boron is irrespective of its chemical bonding state.

Further deactivation experiments were performed at 140, 165 , and $200{ }^{\circ} \mathrm{C}$. Figure 3 depicts the respective deactivation rates $R_{\mathrm{de}}$ of the $1.26 \Omega \mathrm{cm}$ control and the $1.20 \Omega \mathrm{cm}$ compensated wafers in an Arrhenius plot. It can be seen that the deactivation rate of the noncompensated wafer is higher

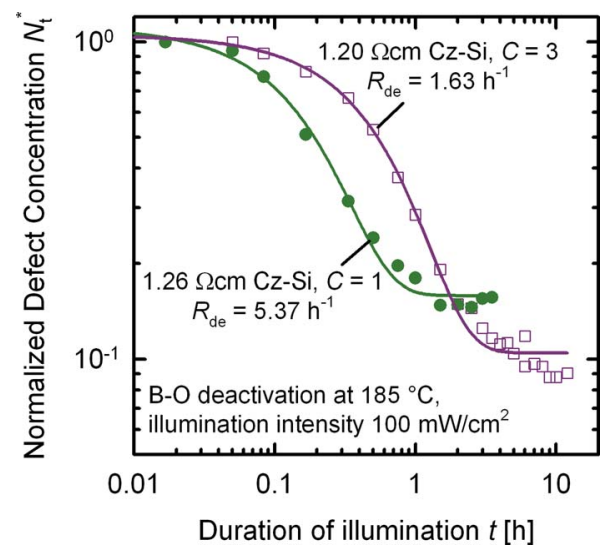

FIG. 1. (Color online) Time dependence of the normalized defect concentration $N_{\mathrm{t}}^{*}$ in two samples during illumination at $185^{\circ} \mathrm{C}$ on a doublelogarithmic scale. The filled green circles refer to a $1.26 \Omega \mathrm{cm}$ boron-doped noncompensated $\mathrm{Cz}-\mathrm{Si}$ wafer, whereas the open purple squares correspond to a $1.20 \Omega \mathrm{cm}$ compensated (B- and P-doped) $\mathrm{Cz}-\mathrm{Si}$ sample. The data is fitted by an exponential decay function which yields the deactivation rate $R_{\mathrm{de}}$. A clear decrease of $R_{\mathrm{de}}$ for the compensated wafer can be seen. 


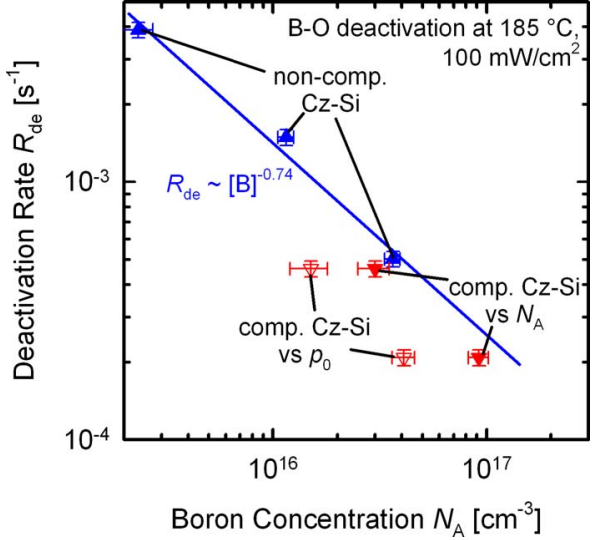

FIG. 2. (Color online) Deactivation rates $R_{\mathrm{de}}$ determined at $185^{\circ} \mathrm{C}$ of noncompensated $\mathrm{Cz}-\mathrm{Si}$ control wafers (filled blue triangles up) are plotted on a double-logarithmic scale vs the total boron concentration $N_{\mathrm{A}}$. The fit with a power law (blue line) yields an exponent of -0.74 . The deactivation rates of the compensated $\mathrm{Cz}-\mathrm{Si}$ wafers are plotted against the net-doping concentration $p_{0}$ (open red triangles down) and the total boron concentration $N_{\mathrm{A}}$ (filled red triangles down). The agreement with the fit is much better for the latter.

than that of the compensated sample at all temperatures. In addition, the fits yield different slopes, which means that they result in two different activation energies $E_{\mathrm{a}}$. So far activation energies between 0.61 (Refs. 4 and 5) and $0.71 \mathrm{eV}$ (Ref. 6) have been reported for silicon with a resistivity of about $1.4 \Omega \mathrm{cm}$. This is slightly less than the value of 0.84 $\mathrm{eV}$ for the $1.26 \Omega \mathrm{cm}$ noncompensated sample found in this study. The activation energy of the $1.20 \Omega \mathrm{cm}$ compensated sample is $1.09 \mathrm{eV}$, however, which is $30 \%$ above the corresponding $E_{\mathrm{a}}$ of the noncompensated reference sample. The $0.45 \Omega \mathrm{cm}$ control yields a comparable value $E_{\mathrm{a}}=1.04 \mathrm{eV}$, whereas the compensated $0.52 \Omega \mathrm{cm} \mathrm{Cz}-\mathrm{Si}$ material has an even higher activation energy of $1.40 \mathrm{eV}$. Plotting the activation energies $E_{\mathrm{a}}$ of all investigated samples as a function of the total boron concentration $N_{\mathrm{A}}$, as depicted in Fig. 4, we find a linear dependence between $E_{\mathrm{a}}$ and $N_{\mathrm{A}}$. The observed linear correlation between $E_{\mathrm{a}}$ and $N_{\mathrm{A}}$ provides additional

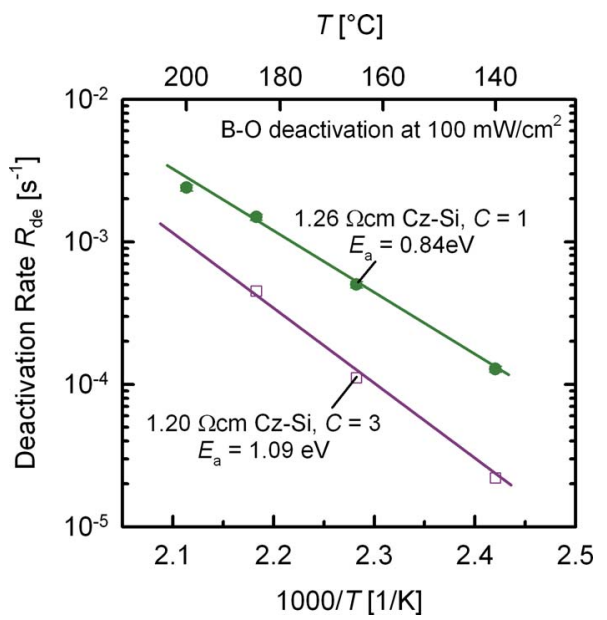

FIG. 3. (Color online) Arrhenius-plot of the deactivation rates $R_{\mathrm{de}}$ of a $1.26 \Omega \mathrm{cm}$ noncompensated $\mathrm{Cz}-\mathrm{Si}$ sample (filled green circles) and a $1.20 \Omega \mathrm{cm}$ compensated $\mathrm{Cz}-\mathrm{Si}$ wafer (open purple squares). The activation energy $E_{\mathrm{a}}$ derived for the compensated wafer is $30 \%$ above that of the control sample.

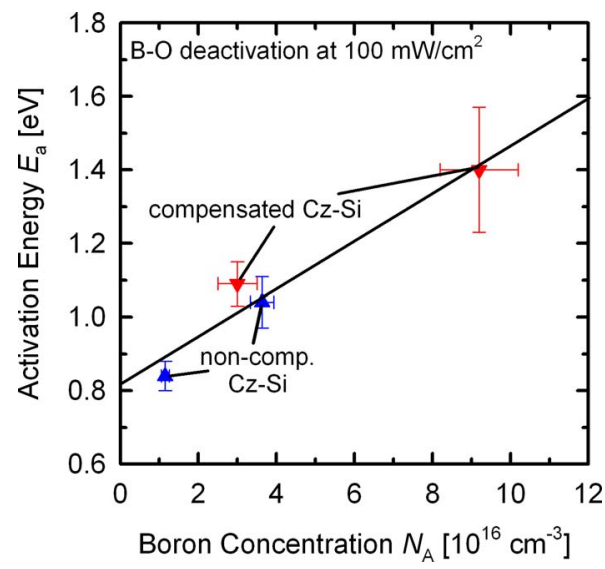

FIG. 4. (Color online) Activation energies $E_{\mathrm{a}}$ of the noncompensated reference wafers (filled blue triangles) and the compensated samples (filled red triangles) plotted vs the total boron concentration $N_{\mathrm{A}}$, showing a linear relationship between $E_{\mathrm{a}}$ and $N_{\mathrm{A}}$.

evidence that boron is directly involved in the deactivation process.

In this study, we have shown that the permanent deactivation of the boron-oxygen-related recombination center also works in compensated silicon. The comparison with noncompensated control wafers revealed that the deactivation rate $R_{\mathrm{de}}$ in compensated silicon depends on the total boron concentration $N_{\mathrm{A}}$ rather than on the hole concentration $p_{0}$. An examination of the activation energy $E_{\mathrm{a}}$ showed a linear correlation of $E_{\mathrm{a}}$ with $N_{\mathrm{A}}$ instead of a correlation with $p_{0}$. Both experimental findings provide strong evidence that boron plays a role in the deactivation process and that its involvement is irrespective of its chemical bonding state.

Funding was provided by the State of Lower Saxony and the DAAD/Go8 Australia Germany Joint Research Cooperation Scheme.

${ }^{1}$ J. Schmidt and K. Bothe, Phys. Rev. B 69, 024107 (2004).

${ }^{2} \mathrm{H}$. Fischer and W. Pschunder, Proceedings of the Tenth IEEE Photovoltaic Specialists Conference, Palo Alto, CA (IEEE, New York, 1973), p. 404.

${ }^{3}$ J. Knobloch, S. W. Glunz, V. Henninger, W. Warta, W. Wettling, F. Schomann, W. Schmidt, A. Endrös, and K. A. Münzer, Proceedings of the 13th European Photovoltaic Solar Energy Conference, Nice, France (Stephens, Bedford, 1995), p. 9.

${ }^{4}$ A. Herguth, G. Schubert, M. Kaes, and G. Hahn, Proceedings of the 21 st European Photovoltaiv Solar Energy Conference, Dresden, Germany (WIP, Munich, 2006), p.530.

${ }^{5}$ A. Herguth, G. Schubert, M. Kaes, and G. Hahn, Prog. Photovoltaics 16, 135 (2008).

${ }^{6}$ B. Lim, K. Bothe, and J. Schmidt, Phys. Status Solidi (RRL) 2, 93 (2008).

${ }^{7}$ D. B. M. Klaassen, Solid-State Electron. 35, 953 (1992).

${ }^{8}$ D. B. M. Klaassen, Solid-State Electron. 35, 961 (1992).

${ }^{9}$ R. Bock, P. P. Altermatt, and J. Schmidt, Proceedings of the 23rd European Photovoltaics Solar Energy Conference, Valencia, Spain (WIP, Munich, 2008), p. 1510.

${ }^{10}$ D. Macdonald, A. Cuevas, and L. J. Geerligs, Appl. Phys. Lett. 92 202119 (2008)

${ }^{11}$ D. Macdonald, M. Kerr, and A. Cuevas, Appl. Phys. Lett. 75, 1571 (1999).

${ }^{12}$ T. Lauinger, J. Schmidt, A. G. Aberle, and R. Hezel, Appl. Phys. Lett. 68, 1232 (1996)

${ }^{13}$ R. Sinton and A. Cuevas, Appl. Phys. Lett. 69, 2510 (1996)

${ }^{14}$ S. Pizzini and C. Calligarich, J. Electrochem. Soc. 131, 2128 (1984).

${ }^{15}$ R. Kopecek, J. Arumughan, K. Peter, E. A. Good, J. Libal, M. Acciarri, and S. Binetti, Proceedings of the 23rd European Photovoltaics Solar Energy Conference, Valencia, Spain (WIP, Munich, 2008), p. 1855.

${ }^{16}$ D. Macdonald, F. Rougieux, A. Cuevas, B. Lim, J. Schmidt, M. Di Sabatino, and L. J. Geerligs, J. Appl. Phys. 105, 093704 (2009). 